\title{
On non-Kupka points of codimension one foliations on $\mathbb{P}^{3}$
}

\author{
OMEGAR CALVO-ANDRADE ${ }^{1}$, MAURÍCIO CÔRREA ${ }^{2}$ and ARTURO FERNÁNDEZ-PÉREZ ${ }^{2}$ \\ ${ }^{1}$ Centro de Investigaciones en Matemáticas/CIMAT, \\ A.C., Jalisco, s/n, Col. Valenciana CP 36023 Guanajuato, Gto, México \\ ${ }^{2}$ Universidade Federal de Minas Gerais, Departamento de Matemática, \\ Av. Antônio Carlos, 6627, 31270-901 Belo Horizonte, MG, Brazil
}

Manuscript received on January 13, 2016; accepted for publication on August 15, 2016

\begin{abstract}
We study the singular set of a codimension one holomorphic foliation on $\mathbb{P}^{3}$. We find a local normal form for these foliations near a codimension two component of the singular set that is not of Kupka type. We also determine the number of non-Kupka points immersed in a codimension two component of the singular set of a codimension one foliation on $\mathbb{P}^{3}$.
\end{abstract}

Key words: holomorphic foliations, Kupka sets, non-Kupka points.

\section{1 - INTRODUCTION}

A regular codimension one holomorphic foliation on a complex manifold $M$, can be defined by a triple $\left\{\left(\mathfrak{U}, f_{\alpha}, \psi_{\alpha \beta}\right)\right\}$ where

1. $\mathfrak{U}=\left\{U_{\alpha}\right\}$ is an open cover of $M$.

2. $f_{\alpha}: U_{\alpha} \rightarrow \mathbb{C}$ is a holomorphic submersion for each $\alpha$.

3. A family of biholomorphisms $\left\{\psi_{\alpha \beta}: f_{\beta}\left(U_{\alpha \beta}\right) \rightarrow f_{\alpha}\left(U_{\alpha \beta}\right)\right\}$ such that

$$
\psi_{\alpha \beta}=\psi_{\beta \alpha}^{-1},\left.\quad f_{\beta}\right|_{U_{\alpha} \cap U_{\beta}}=\left.\psi_{\beta \alpha} \circ f_{\alpha}\right|_{U_{\alpha} \cap U_{\beta}} \text { and } \quad \psi_{\alpha \gamma}=\psi_{\alpha \beta} \circ \psi_{\beta \gamma} \text {. }
$$

AMS(1991): 37F75, 32S65

Correspondence to: Maurício Côrrea

E-mail: mauriciomatufmg@gmail.com

Dedicated to José Seade in his 60 birthday. 
Since $d f_{\alpha}(x)=\psi_{\alpha \beta}^{\prime}\left(f_{\beta}(x)\right) \cdot d f_{\beta}(x)$, the set $F=\bigcup_{\alpha} \operatorname{Ker}\left(d f_{\alpha}\right) \subset T M$ is a subbundle. Also $\left[\psi_{\alpha \beta}^{\prime}\left(f_{\beta}\right)\right] \in$ $\check{H}^{1}\left(\mathfrak{U}, \mathcal{O}^{*}\right)$ define a line bundle $N=T M / F$. The family of 1 -forms $\left\{d f_{\alpha}\right\}$ glue to a global section $\omega \in$ $H^{0}\left(M, \Omega^{1}(N)\right)$. We have

$$
0 \rightarrow F \rightarrow T M \stackrel{\left\{d f_{\alpha}\right\}}{\longrightarrow} N \rightarrow 0, \quad 0 \rightarrow \mathcal{F} \rightarrow \Theta \stackrel{\left\{d f_{\alpha}\right\}}{\longrightarrow} \mathcal{N} \rightarrow 0, \quad[\mathcal{F}, \mathcal{F}] \subset \mathcal{F}
$$

where $\mathcal{F}=\mathcal{O}(F), \Theta=\mathcal{O}(T M)$ and $\mathcal{N}=\mathcal{O}(N)$. We also obtain

$$
\wedge^{n} T M^{*}=\operatorname{det}\left(F^{*}\right) \otimes N^{*}, \quad \Omega_{M}^{n}:=K_{M}=\operatorname{det}\left(\mathcal{F}^{*}\right) \otimes \mathcal{N}^{*}, \quad n=\operatorname{dim}(M) .
$$

Definition 1.1. Let $M$ be a compact complex manifold of dimension $n$. A singular codimension one holomorphic foliation on $M$, may be defined by one of the following ways:

1. A pair $\mathcal{F}=(S, \mathcal{F})$, where $S \subset M$ is an analytic subset of $\operatorname{codim}(S) \geq 2$, and $\mathcal{F}$ is a regular codimension one holomorphic foliation on $M \backslash S$.

2. A class of global sections $[\omega] \in \mathbb{P} H^{0}\left(M, \Omega^{1}(L)\right)$, where $L \in \operatorname{Pic}(M)$ such that

(a) the singular set $S_{\omega}=\left\{p \in M \mid \omega_{p}=0\right\}$ has $\operatorname{codim}\left(S_{\omega}\right) \geq 2$.

(b) $\omega \wedge d \omega=0$ in $H^{0}\left(M, \Omega^{3}\left(L^{\otimes 2}\right)\right)$.

In this case, we denote by $\mathcal{F}_{\omega}=\left(S_{\omega}, \mathcal{F}_{\omega}\right)$ the foliation represented by $\omega$.

3. An exact sequence of sheaves

$$
0 \rightarrow \mathcal{F} \rightarrow \Theta \rightarrow \mathcal{N} \rightarrow 0, \quad[\mathcal{F}, \mathcal{F}] \subset \mathcal{F}
$$

where $\mathcal{F}$ is a reflexive sheaf of rank $r k(\mathcal{F})=n-1$ with torsion free quotient $\mathcal{N} \simeq \mathcal{J}_{S} \otimes L$, where $\mathcal{J}_{S}$ is an ideal sheaf for some closed scheme $S$.

These three definitions are equivalents.

Remark 1.2. Let $\omega \in H^{0}\left(M, \Omega^{1}(L)\right)$ be a section.

1. The section $\omega$ may be defined by a family of 1 -forms

$$
\omega_{\alpha} \in \Omega^{1}\left(U_{\alpha}\right), \quad \omega_{\alpha}=\lambda_{\alpha \beta} \omega_{\beta} \text { in } U_{\alpha \beta}=U_{\alpha} \cap U_{\beta}, \quad L=\left[\lambda_{\alpha \beta}\right] \in \check{H}^{1}\left(\mathfrak{U}, \mathcal{O}^{*}\right) .
$$

2. The section $\omega$ is a morphism of sheaves $\Theta \stackrel{\omega}{\longrightarrow} L$. The kernel of $\omega$ is the tangent sheaf $\mathcal{F}$. The image of $\omega$ is a twisted ideal sheaf $\mathcal{N}=\mathcal{J}_{S_{\omega}} \otimes L$. It is called the normal sheaf of $\mathcal{F}$.

3. As in the non-singular case, the following equality of line bundles holds

$$
K_{M}=\Omega_{M}^{n}=\operatorname{det}\left(\mathcal{F}^{*}\right) \otimes \mathcal{N}^{*}=K_{\mathcal{F}} \otimes L^{-1}, \quad \operatorname{det}(N) \simeq L
$$

where $K_{M}, K_{\mathcal{F}}=\operatorname{det}\left(\mathcal{F}^{*}\right)$ are the canonical sheaf of $M$ and $\mathcal{F}$ respectively.

We denote by

$$
\begin{aligned}
\mathcal{F}(M, L) & =\left\{[\omega] \in \mathbb{P} H^{0}\left(M, \Omega^{1}(L)\right) \mid \operatorname{codim}\left(S_{\omega}\right) \geq 2, \quad \omega \wedge d \omega=0\right\} \\
\mathcal{F}(n, d) & =\left\{[\omega] \in \mathbb{P} H^{0}\left(\mathbb{P}^{n}, \Omega^{1}(d+2)\right) \mid \operatorname{codim}\left(S_{\omega}\right) \geq 2, \quad \omega \wedge d \omega=0\right\} .
\end{aligned}
$$

The number $d \geq 0$ is called the degree of the foliation represented by $\omega$. 


\section{1 - STATEMENT OF THE RESULTS}

In the sequel, $M$ is a compact complex manifold with $\operatorname{dim}(M) \geq 3$. We will use any of the above definitions for foliation. The singular set will be denoted by $S$. Observe that $S$ decomposes as

$$
S=\bigcup_{k=2}^{n} S_{k} \quad \text { where } \operatorname{codim}\left(S_{k}\right)=k .
$$

For a foliation $\mathcal{F}$ on $M$ represented by $\omega \in \mathcal{F}(M, L)$, the Kupka set (Kupka 1964, De Medeiros 1977) is defined by

$$
K(\omega)=\{p \in M \mid \omega(p)=0, d \omega(p) \neq 0\} .
$$

We recall that for points near $K(\omega)$ the foliation $\mathcal{F}$ is biholomorphic to a product of a dimension one foliation in a transversal section by a regular foliation of codimension two (Kupka 1964) and in particular we have $K(\omega) \subset S_{2}$.

In this note, we focus our attention on the set of non-Kupka points $N K(\omega)$ of $\omega$. The first remark is

$$
N K(\omega)=\{p \in M \mid \omega(p)=0, d \omega(p)=0\} \supset S_{3} \cup \cdots \cup S_{n} .
$$

We analyze three cases, one in each section, the last two being the core of the work.

1. $S_{2}=K(\omega)$, then $N K(\omega)=S_{3} \cup \cdots \cup S_{n}$.

2. There is an irreducible component $Z \subset S_{2}$ such that $Z \cap K(\omega)=\emptyset$.

3. For a foliation $\omega \in \mathcal{F}(3, d)$. Let $Z \subset S_{2}$ be a connected component such that $Z \backslash Z \cap K(\omega)$ is a finite set of points.

The first case has been considered in Brunella (2009), Calvo-Andrade (1999, 2016), Calvo-Andrade and Soares (1994), Cerveau and Lins Neto (1994). Let $\omega \in \mathcal{F}(n, d)$ be a foliation with $K(\omega)=S_{2}$ and connected, then $\omega$ has a meromorphic first integral. In the generic case, the leaves define a Lefschetz or $a$ Branched Lefschetz Pencil. The non-Kupka points are isolated singularities $N K(\omega)=S_{n}$. In this note, we present a new and short proof of this fact when the transversal type of $K(\omega)$ is radial.

In the second section, we study the case of a non-Kupka irreducible component of $S_{2}$. These phenomenon arise naturally in the intersection of irreducible components of $\mathcal{F}(M, L)$. The following result is a local normal form for $\omega$ near the singular set and is a consequence of a result of Loray (2006).

Theorem 1. Let $\omega \in \Omega^{1}\left(\mathbb{C}^{n}, 0\right), n \geq 3$, be a germ of integrable 1 -form such that codim $\left(S_{\omega}\right)=2,0 \in S_{\omega}$ is a smooth point and $d \omega=0$ on $S_{\omega}$. If $j_{0}^{1} \omega \neq 0$, then or either

1. there exists a coordinate system $\left(x_{1}, \ldots, x_{n}\right) \in \mathbb{C}^{n}$ such that

$$
j_{0}^{1}(\omega)=x_{1} d x_{2}+x_{2} d x_{1}
$$

and $\mathcal{F}_{\omega}$ is biholomorphic to the product of a dimension one foliation in a transversal section by a regular foliation of codimension two, or 
2. there exists a coordinate system $\left(x_{1}, \ldots, x_{n}\right) \in \mathbb{C}^{n}$ such that

$$
\omega=x_{1} d x_{1}+g_{1}\left(x_{2}\right)\left(1+x_{1} g_{2}\left(x_{2}\right)\right) d x_{2},
$$

such that $g_{1}, g_{2} \in \mathcal{O}_{\mathbb{C}, 0}$ with $g_{1}(0)=g_{2}(0)=0$, or

3. $\omega$ has a non-constant holomorphic first integral in a neighborhood of $0 \in \mathbb{C}^{n}$.

The alternatives are not exclusives. The following example was suggest by the referee and show that the case (3) of Theorem 1 cannot be avoid.

Example 1.3. Let $\omega$ be a germ of a 1 -form at $0 \in \mathbb{C}^{3}$ defined by

$$
\omega=x d x+(1+x f) d f
$$

where $f(x, y, z)=y^{2} z$. We have

$$
\omega=x d x+2 y z\left(1+x y^{2} z\right) d y+y^{2}\left(1+x y^{2} z\right) d z .
$$

The singular set of $\omega$ is $\{x=y=0\}$ and $\left\{x=z=y^{2}=0\right\}$, therefore the singular set has an embedding point $\left\{x=z=y^{2}=0\right\}$ and $d \omega$ vanish along $\{x=y=0\}$. We will show that $\omega$ has a holomorphic first integral $F$ in a neighborhood of $0 \in \mathbb{C}^{3}$. In fact, let $t=f(x, y, z)=y^{2} z$ and set $\varphi:\left(\mathbb{C}^{3}, 0\right) \rightarrow\left(\mathbb{C}^{2}, 0\right)$ defined by

$$
\varphi(x, y, z)=(x, t) .
$$

Let $\eta=x d x+(1+x t) d t$ be 1 -form at $0 \in \mathbb{C}^{2}$, note that $\omega=\varphi^{*}(\eta)$ and moreover $\eta(0,0) \neq 0$, this implies that $\eta$ is non-singular at $0 \in \mathbb{C}^{2}$ and by Frobenius theorem $\eta$ has a holomorphic first integral $H(x, t)$ on $\left(\mathbb{C}^{2}, 0\right)$. Defining $H_{1}(x, y, z):=H(x, f(x, y, z))=H\left(x, y^{2} z\right)$, we get $H_{1}$ is a holomorphic first integral for $\omega$ in a neighborhood of $0 \in \mathbb{C}^{3}$.

We apply Theorem 1 to a codimension one holomorphic foliation of the projective space with empty Kupka set.

About the third case, consider a foliation $\omega \in \mathcal{F}(3, d)$. Let $Z$ be a connected component of $S_{2}$. We count the number $|Z \cap N K(\omega)|$ of non-Kupka points of $\omega$ in $Z \subset S_{2}$.

Theorem 2. Let $\omega \in \mathcal{F}(3, d)$ be a foliation and $Z \subset S_{2}$ a connected component of $S_{2}$. Suppose that $Z$ is a local complete intersection and $Z \backslash Z \cap K(\omega)$ is a finite set of points, then $\left.d \omega\right|_{Z}$ is a global section of $\left.K_{Z}^{-1} \otimes K_{\mathcal{F}}\right|_{Z}$ and the associated divisor $D_{\omega}=\sum_{p \in Z} \operatorname{ord}_{p}(d \omega) \cdot p$ has degree

$$
\operatorname{deg}\left(D_{\omega}\right)=\operatorname{deg}\left(K_{\mathcal{F}}\right)-\operatorname{deg}\left(K_{Z}\right)
$$

Note that the section $\left.d \omega\right|_{Z}$ vanishes exactly in the non-Kupka points of $\omega$ in $Z$ then the above theorem determine the number $|Z \cap N K(\omega)|$ (counted with multiplicity) of non-Kupka points of $\omega$ in $Z$.

\section{2 - THE SINGULAR SET}

Let $\omega \in \mathcal{F}(M, L)$ be a codimension one holomorphic foliation then singular set of $\omega$ may be written as

$$
S=\bigcup_{j=2}^{n} S_{j} \quad \text { where } \quad \operatorname{codim}\left(S_{j}\right)=j .
$$


The fact that $K(\omega) \subset S_{2}$ implies that $S_{3} \cup \ldots \cup S_{n} \subset N K(\omega)$. To continue we focus in the components of singular set of $\omega$ of dimension at least three.

\section{1 - SINGULAR SET OF CODIMENSION AT LEAST THREE}

We recall the following result due to B. Malgrange.

Theorem 2.1. (Malgrange 1976) Let $\omega$ be a germ at $0 \in \mathbb{C}^{n}, n \geq 3$ of an integrable 1-form singular at 0 , if $\operatorname{codim}\left(S_{\omega}\right) \geq 3$, then there exist $f \in \mathcal{O}_{\mathbb{C}^{n}, 0}$ and $g \in \mathcal{O}_{\mathbb{C}^{n}, 0}^{*}$ such that

$$
\omega=g d f \quad \text { on a neighborhood of } 0 \in \mathbb{C}^{n} \text {. }
$$

We have the following proposition.

Proposition 2.2. Let $\omega \in \mathcal{F}(M, L)$ be a foliation and let $p \in S_{n}$ an isolated singularity, then any germ of vector field tangent to the foliation vanishes at $p$.

Proof. Let $\omega=g d f, \quad g \in \mathcal{O}_{p}^{*}, f \in \mathcal{O}_{p}$ be a 1-form representing the foliation at $p$. Let $\mathbf{X} \in \Theta_{p}$ be a vector field tangent to the foliation, i.e., $\omega(\mathbf{X})=0$. If $\mathbf{X}(p) \neq 0$ there exists a coordinate system with $z(p)=0$ and $\mathbf{X}=\partial / \partial z_{n}$, then

$$
0=\omega(\mathbf{X})=g \cdot\left(\sum_{i=1}^{n}\left(\partial f / \partial z_{i}\right) d z_{i}\left(\partial / \partial z_{n}\right)\right)=g \cdot\left(\partial f / \partial z_{n}\right), \text { therefore } \partial f / \partial z_{n} \equiv 0
$$

and $f=f\left(z_{1}, \ldots, z_{n-1}\right)$, but this function does not have an isolated singularity.

Now, we begin our study of the irreducible components of codimension two of the singular set of $\omega$. Note that, given a section $\omega \in H^{0}\left(M, \Omega^{1}(L)\right)$, along the singular set, the equation $\omega_{\alpha}=\lambda_{\alpha \beta} \omega_{\beta}$ implies $\left.d \omega_{\alpha}\right|_{S}=\left.\left(\lambda_{\alpha \beta} d \omega_{\beta}\right)\right|_{S}$. Then

$$
\left\{d \omega_{\alpha}\right\} \in H^{0}\left(S,\left.\left(\Omega_{M}^{2} \otimes L\right)\right|_{S}\right)
$$

\section{2 - THE KUPKA SET}

These singularities has bee extensively studied and the main properties have been established in (Kupka 1964, De Medeiros 1977).

Definition 2.3. For $\omega \in \mathcal{F}(M, L)$. The Kupka set is

$$
K(\omega)=\{p \in M \mid \omega(p)=0, \quad d \omega(p) \neq 0\} .
$$

The following properties of Kupka sets, are well known (De Medeiros 1977).

1. $K(\omega)$ is smooth of codimension two.

2. $K(\omega)$ has local product structure and the tangent sheaf $\mathcal{F}$ is locally free near $K(\omega)$.

3. $K(\omega)$ is subcanonically embedded and

$$
\wedge^{2} N_{K(\omega)}=\left.L\right|_{K(\omega)}, \quad K_{K(\omega)}=\left.\left(K_{M} \otimes L\right)\right|_{K(\omega)}=\left.K_{\mathcal{F}}\right|_{K(\omega)} .
$$


Let $\omega \in \mathcal{F}(n, d)$ be a foliation with $S_{2}=K(\omega)$. By Calvo-Andrade and Soares (1994), there exists a pair $(V, \sigma)$, where $V$ is a rank two holomorphic vector bundle and $\sigma \in H^{0}\left(\mathbb{P}^{n}, V\right)$, such that

$$
0 \longrightarrow \mathcal{O} \stackrel{\sigma}{\longrightarrow} V \longrightarrow \mathcal{J}_{K}(d+2) \rightarrow 0 \quad \text { with } \quad\{\sigma=0\}=K
$$

and the total Chern class

$$
c(V)=1+(d+2) \cdot \mathbf{h}+\operatorname{deg}(K(\omega)) \mathbf{h}^{2} \in H^{*}\left(\mathbb{P}^{n}, \mathbb{Z}\right) \simeq \mathbb{Z}[\mathbf{h}] / \mathbf{h}^{n+1} .
$$

In 2009, Marco Brunella proved that following result, which in a certain sense say that the local transversal type of the singular set of foliation determines its behavior globally. Here we present a new proof of this fact. The techniques used in the proof could be of independent interest.

Proposition 2.4. Let $\omega \in \mathcal{F}(n, d)$ be a foliation with $S_{2}=K(\omega)$, (connected if $n=3$ ) and of radial transversal type. Then $K(\omega)$ is a complete intersection and $\omega$ has a meromorphic first integral.

To prove Proposition 2.4, we requires the following lemma. This result may be well known but for lack of a suitable reference we include the proof in an appendix.

Lemma 2.5. Let $F$ be a rank two holomorphic vector bundle over $\mathbb{P}^{2}$ with $c_{1}(F)=0$ and $c_{2}(F)=0$. Then $F \simeq \mathcal{O} \oplus \mathcal{O}$, is holomorphically trivial.

Now, we prove Proposition 2.4.

Proof of Proposition 2.4. Let $(V, \sigma)$ be the vector bundle with a section defining the Kupka set as scheme. The radial transversal type implies (Calvo-Andrade and Soares 1994)

$$
c(V)=1+(d+2) \cdot \mathbf{h}+\frac{(d+2)^{2}}{4} \cdot \mathbf{h}^{2}=\left(1+\frac{(d+2) \cdot \mathbf{h}}{2}\right)^{2} \in H^{*}\left(\mathbb{P}^{n}, \mathbb{Z}\right) \simeq \mathbb{Z}[\mathbf{h}] / \mathbf{h}^{n+1} .
$$

The vector bundle $E=V\left(-\frac{d+2}{2}\right)$, has $c_{1}(E)=0$ and $c_{2}(E)=0$. Let $\xi: \mathbb{P}^{2} \hookrightarrow \mathbb{P}^{n}$ be a linear embedding. By the preceding lemma we have

$$
\xi^{*} E \simeq \mathcal{O}_{\mathbb{P}^{2}} \oplus \mathcal{O}_{\mathbb{P}^{2}}
$$

and by the Horrocks' criterion (Okonek et al. 1980),

$$
E \simeq \mathcal{O}_{\mathbb{P}^{n}} \oplus \mathcal{O}_{\mathbb{P}^{n}}
$$

is trivial and hence $V$ splits as $\mathcal{O}_{\mathbb{P}^{n}}\left(\frac{d+2}{2}\right) \oplus \mathcal{O}_{\mathbb{P}^{n}}\left(\frac{d+2}{2}\right)$ and $K$ is a complete intersection. The existence of the meromorphic first integral follows from Theorem A of (Cerveau and Lins Neto 1994).

If $\omega$ is such that $K(\omega)=S_{2}$ and connected, the set of non-Kupka points of $\omega$ is

$$
N K(\omega)=S_{3} \cup \cdots \cup S_{n} .
$$

A generic rational map, that means, a Lefschetz or a Branched Lefschetz Pencil $\varphi: \mathbb{P}^{n} \rightarrow \mathbb{P}^{1}$, has only isolated singularities away its base locus. The singular set of the foliation defined by the fibers of $\varphi$ is $S_{n} \cup S_{2}$. The Kupka set corresponds away from its base locus and $S_{n}=N K(\omega)$ are the singularities as a map. $S_{n}$ is empty if and only if the degree of the foliation is 0 . The number $\ell\left(S_{n}\right)$ of isolated singularities 
counted with multiplicities can be calculated by (Cukierman et al. 2006). If $\omega_{p}$ is a germ of form that defines the foliation at $p \in S_{n}$, we have

$$
\ell\left(S_{n}\right)=\sum_{p \in S_{n}} \mu\left(\omega_{p}, p\right), \quad \mu(\omega, p)=\operatorname{dim}_{\mathbb{C}} \frac{\mathcal{O}_{p}}{\left(\omega_{1}, \ldots, \omega_{n}\right)}, \quad \omega_{p}=\sum_{i=1}^{n} \omega_{i} d z_{i} .
$$

We have that $c_{n}(\mathcal{F})=\ell\left(S_{n}\right)$.

\section{3 - FOLIATIONS WITH A NON-KUPKA COMPONENT}

It is well known that $K(\omega) \subset\left\{p \in M \mid j_{p}^{1} \omega \neq 0\right\}$, but the converse is not true. Our first result describes the singular points with this property.

\section{1 - A NORMAL FORM}

Now, we analyze the situation when there is an irreducible non-Kupka component of $S_{2}$.

Proof of Theorem 1. By hypotheses, $d \omega(p)=0$ for any $p \in S_{\omega}$. Since

$$
\omega=\omega_{1}+\cdots, \quad d \omega=d \omega_{1}+\cdots=0,
$$

we get $d \omega_{1}(p)=0$ for any $p \in S_{\omega}$. Now, as $\omega_{1} \neq 0$ and $\operatorname{codim}\left(S_{\omega}\right)=2$, we have $1 \leq \operatorname{codim}\left(S_{\omega_{1}}\right) \leq 2$. We distinguish two cases.

1. $\operatorname{codim}\left(S_{\omega_{1}}\right)=2$ : there is a coordinate system $\left(x_{1}, \ldots, x_{n}\right) \in \mathbb{C}^{n}$ such that

$$
\omega_{1}=x_{1} d x_{2}+x_{2} d x_{1} .
$$

2. $\operatorname{codim}\left(S_{\omega_{1}}\right)=1$ : there is a coordinate system $(x, \zeta) \in \mathbb{C} \times \mathbb{C}^{n-1}$ such that $x(p)=0$ and $\omega_{1}=x d x$.

The first case is known, the foliation $\mathcal{F}_{\omega}$ is equivalent in a neighborhood of $0 \in \mathbb{C}^{n}$ to a product of a dimension one foliation in a transversal section by a regular foliation of codimension two (Cerveau and Mattei 1982).

In the second case, Loray's preparation theorem (Loray 2006), shows that there exists a coordinate system $(x, \zeta) \in \mathbb{C} \times \mathbb{C}^{n-1}$, a germ $f \in \mathcal{O}_{\mathbb{C}^{n-1}, 0}$ with $f(0)=0$, and germs $g, h \in \mathcal{O}_{\mathbb{C}, 0}$ such that the foliation is defined by the 1 -form

$$
\omega=x d x+[g(f(\zeta))+x h(f(\zeta))] d f(\zeta) .
$$

Since $S_{\omega_{1}}=\{x=0\}$ and $0 \in S_{\omega}$ is a smooth point, we can assume that $S_{\omega, p}=\left\{x=\zeta_{1}=0\right\}$, where $S_{\omega, p}$ is the germ of $S_{\omega}$ at $p=0$. Therefore,

$$
S_{\omega, p}=\left\{x=\zeta_{1}=0\right\}=\{x=g(f(\zeta))=0\} \cup\left\{x=\frac{\partial f}{\partial \zeta_{1}}=\cdots=\frac{\partial f}{\partial \zeta_{n-1}}=0\right\} .
$$

Hence, either $g(0)=0$ and $\zeta_{1} \mid f$, or $g(0) \neq 0$ and $\zeta_{1} \mid \frac{\partial f}{\partial \zeta_{j}}$ for all $j=1, \ldots, n-1$. In any case, we have $\zeta_{1} \mid f$ and then $f(\zeta)=\zeta_{1}^{k} \psi(\zeta)$, where $\psi$ is a germ of holomorphic function in the variable $\zeta ; k \in \mathbb{N}$ and $\zeta_{1}$ does not divide $\psi$. We have two possibilities: 
$1^{\text {st }}$ case. $-\psi(0) \neq 0$. In this case, we consider the biholomorphism

$$
G(x, \zeta)=\left(x, \zeta_{1} \psi^{1 / k}(\zeta), \zeta_{2}, \ldots, \zeta_{n}\right)=\left(x, y, \zeta_{2}, \ldots, \zeta_{n}\right)
$$

where $\psi^{1 / k}$ is a branch of the $k^{t h}$ root of $\psi$, we get $f \circ G^{-1}\left(x, y, \zeta_{2}, \ldots, \zeta_{n}\right)=y^{k}$ and

$$
G_{*}(\omega)=x d x+\left(g\left(y^{k}\right)+x h\left(y^{k}\right)\right) k y^{k-1} d y=x d x+\left(g_{1}(y)+x h_{1}(y)\right) d y,
$$

where $g_{1}(y)=k y^{k-1} g\left(y^{k}\right), h_{1}(y)=k y^{k-1} h\left(y^{k}\right)$. Therefore, $\tilde{\omega}:=G_{*}(\omega)$ is equivalent to $\omega$ and moreover $\tilde{\omega}$ is given by

$$
\tilde{\omega}=x d x+\left(g_{1}(y)+x h_{1}(y)\right) d y \quad \text { with } \quad S_{\tilde{\omega}}=\left\{x=g_{1}(y)=0\right\} .
$$

Since $d \tilde{\omega}=h_{1}(y) d x \wedge d y$ is zero identically on $\left\{x=g_{1}(y)=0\right\}$, we get $g_{1} \mid h_{1}$, so that $h_{1}(y)=$ $\left(g_{1}(y)\right)^{m} H(y)$, for some $m \in \mathbb{N}$ and such that $H(y)$ does not divided $g_{1}(y)$. Using the above expression for $h_{1}$ in (3.2), we have

$$
\tilde{\omega}=x d x+g_{1}(y)\left(1+x\left(g_{1}(y)\right)^{m-1} H(y)\right) d y=x d x+g_{1}(y)\left(1+x g_{2}(y)\right) d y,
$$

where $g_{2}(y)=\left(g_{1}(y)\right)^{m-1} H(y)$. Consider $\varphi:(\mathbb{C}, 0) \times\left(\mathbb{C}^{n-1}, 0\right) \rightarrow\left(\mathbb{C}^{2}, 0\right)$ defined by $\varphi(x, \zeta)=(x, y)$, then

$$
\omega=\varphi^{*}\left(x d x+g_{1}(y)\left(1+x g_{2}(y)\right) d y\right) .
$$

$2^{n d}$ case. $-\psi(0)=0$. We have $S_{\omega, p}=\left\{x=\zeta_{1}=0\right\}$ and

$$
\omega=x d x+\left(g\left(\zeta_{1}^{k} \psi\right)+x h\left(\zeta_{1}^{k} \psi\right)\right) d\left(\zeta_{1}^{k} \psi\right)
$$

therefore

$$
\omega=x d x+\left(g\left(\zeta_{1}^{k} \psi\right)+x h\left(\zeta_{1}^{k} \psi\right)\right) \zeta_{1}^{k-1}\left(k \psi d \zeta_{1}+\zeta_{1} d \psi\right)
$$

Note that $g(0) \neq 0$, otherwise $\left\{x=\zeta_{1} \psi(\zeta)=0\right\}$ would be contained in $S_{\omega, p}$, but it is contradiction because $S_{\omega, p}=\left\{x=\zeta_{1}=0\right\} \subsetneq\left\{x=\zeta_{1} \psi(\zeta)=0\right\}$. Furthermore $k \geq 2$, because otherwise $\zeta_{1} \mid \psi$.

Let $\varphi:(\mathbb{C}, 0) \times\left(\mathbb{C}^{n-1}, 0\right) \rightarrow\left(\mathbb{C}^{2}, 0\right)$ be defined by

$$
\varphi(x, \zeta)=\left(x, \zeta_{1}^{k} \psi(\zeta)\right)=(x, t)
$$

then from (3.4), we get that

$$
\omega=\varphi^{*}(\eta)
$$

where $\eta=x d x+(g(t)+x h(t)) d t$. Since $\eta(0,0)=g(0) d t \neq 0$, we deduce that $\eta$ has a non-constant holomorphic first integral $F \in \mathcal{O}_{\mathbb{C}^{2}, 0}$ such that $d F(0,0) \neq 0$. Therefore, $F_{1}(x, \zeta)=F\left(x, \zeta_{1}^{k} \psi(\zeta)\right)$ is a non-constant holomorphic first integral for $\omega$ in a neighborhood of $0 \in \mathbb{C}^{n}$.

\section{2 - APPLICATIONS TO FOLIATIONS ON $\mathbb{P}^{n}$}

In order to give some applications of Theorem 1, we need the Baum-Bott index associated to singularities of foliations of codimension one.

Let $M$ be a complex manifold and let $\mathcal{G}_{\omega}=(S, \mathcal{G})$ be a codimension one holomorphic foliation represented by $\omega \in H^{0}\left(M, \Omega^{1}(L)\right)$. We have the exact sequence

$$
0 \rightarrow \mathcal{G} \rightarrow \Theta_{M} \stackrel{\omega}{\rightarrow} \mathcal{N}_{\mathcal{G}} \rightarrow 0, \quad \mathcal{N}_{\mathcal{G}} \simeq \mathcal{J}_{S} \otimes L
$$


Set $M^{0}=M \backslash S$ and take $p_{0} \in M^{0}$. Then in a neighborhood $U_{\alpha}$ of $p_{0}$ the foliation $\mathcal{G}$ is induced by a holomorphic 1 -form $\omega_{\alpha}$ and there exists a differentiable 1 -form $\theta_{\alpha}$ such that

$$
d \omega_{\alpha}=\theta_{\alpha} \wedge \omega_{\alpha}
$$

Let $Z$ be an irreducible component of $S_{2}$. Take a generic point $p \in Z$, that is, $p$ is a point where $Z$ is smooth and disjoint from the other singular components. Pick $B_{p}$ a ball centered at $p$ sufficiently small, so that $S\left(B_{p}\right)$ is a sub-ball of $B_{p}$ of codimension 2 . Then the De Rham class can be integrated over an oriented 3-sphere $L_{p} \subset B_{p}^{*}$ positively linked with $S\left(B_{p}\right)$ :

$$
\mathrm{BB}(\mathcal{G}, Z)=\frac{1}{(2 \pi i)^{2}} \int_{L_{p}} \theta \wedge d \theta .
$$

This complex number is the Baum-Bott residue of $\mathcal{G}$ along $Z$. We have a particular case of the general Baum-Bott residues Theorem (Baum and Bott 1972), reproved by Brunella and Perrone (2011).

Theorem 3.1. Let $\mathcal{G}$ be a codimension one holomorphic foliation on a complex manifold $M$. Then

$$
c_{1}(L)^{2}=c_{1}^{2}\left(\mathcal{N}_{\mathcal{G}}\right)=\sum_{Z \subset S_{2}} B B(\mathcal{G}, Z)[Z]
$$

where $\mathcal{N}_{\mathcal{G}}=\mathcal{J}_{S} \otimes L$ is the normal sheaf of $\mathcal{G}$ on $M$ and the sum is done over all irreducible components of $S_{2}$.

In particular, if $\mathcal{G}$ is a codimension one foliation on $\mathbb{P}^{n}$ of degree $d$, then the normal sheaf $\mathcal{N}_{\mathcal{G}}=$ $\mathcal{J}_{S}(d+2)$ and the Baum-Bott Theorem looks as follows

$$
\sum_{Z \subset S_{2}} \operatorname{BB}(\mathcal{G}, Z) \operatorname{deg}[Z]=(d+2)^{2} .
$$

Remark 3.2. If there exist a coordinates system $\left(U,\left(x, y, z_{3}, \ldots, z_{n}\right)\right)$ around $p \in Z \subset S_{2}$ such that $x(p)=y(p)=0$ and $S(\mathcal{G}) \cap U=Z \cap U=\{x=y=0\}$. Moreover, if we assume that

$$
\left.\omega\right|_{U}=P(x, y) d y-Q(x, y) d x
$$

is a holomorphic 1-form representing $\left.\mathcal{G}\right|_{U}$. Then we can consider the $\mathcal{C}^{\infty}(1,0)$-form $\theta$ on $U \backslash Z$ given by

$$
\theta=\frac{\left(\frac{\partial P}{\partial x}+\frac{\partial Q}{\partial y}\right)}{|P|^{2}+|Q|^{2}}(\bar{P} d x+\bar{Q} d y)
$$

Since $d \omega=\theta \wedge \omega$, we get

$$
\mathrm{BB}(\mathcal{G}, Z)=\frac{1}{(2 \pi i)^{2}} \int_{L_{p}} \theta \wedge d \theta=\operatorname{Res}_{0}\left\{\frac{\operatorname{Tr}(D \mathbf{X}) d x \wedge d y}{P Q}\right\},
$$

where $\operatorname{Res}_{0}$ denotes the Grothendieck residue, $D \mathbf{X}$ is the Jacobian of the holomorphic map $\mathbf{X}=(P, Q)$. It follows from Griffiths and Harris (1978) that if $D \mathbf{X}(p)$ is non-singular, then

$$
\operatorname{BB}(\mathcal{G}, Z)=\frac{\operatorname{Tr}(D \mathbf{X}(p))^{2}}{\operatorname{det}(D \mathbf{X}(p))} .
$$


In the situation explained above, the tangent sheaf $\mathcal{G}(U)$ is locally free and generated by the holomorphic vector fields

$$
\mathcal{G}(U)=\left\langle\mathbf{X}=P(x, y) \frac{\partial}{\partial x}+Q(x, y) \frac{\partial}{\partial y}, \frac{\partial}{\partial z_{3}}, \ldots, \frac{\partial}{\partial z_{n}}\right\rangle
$$

and the vector field $\mathbf{X}$ carries the information of the Baum-Bott residues.

The next result, in an application of Theorem 1

Theorem 3.3. Let $\omega \in \mathcal{F}(M, L)$ be a foliation and $Z \subset S_{2} \backslash K(\omega)$. Suppose that $Z$ is smooth and $j_{p}^{1} \omega \neq 0$ for all $p \in Z$, then $B B\left(\mathcal{F}_{\omega}, Z\right)=0$.

Proof. We work in a small neighborhood $U$ of $p \in Z \subset M$. According to Theorem 1 there exist a coordinate system $\left(x, y, z_{3}, \ldots, z_{n}\right)$ at $p$ such that $Z \cap U=\{x=y=0\}$ and one has three cases. In the first case, $\mathcal{F}_{\omega}$ is the product of a dimension one foliation in a section transversal to $Z$ by a regular foliation of codimension two and $j_{p}^{1}(\omega)=x d y+y d x$. In this case, it follows from (3.6) that $\operatorname{BB}\left(\mathcal{F}_{\omega}, Z\right)=0$. In the second case

$$
\omega=x d x+g_{1}(y)\left(1+x g_{2}(y)\right) d y
$$

where $g_{1}, g_{2} \in \mathcal{O}_{\mathbb{C}, 0}$ and it follows from Lemma 3.9 of Cerveau and Lins Neto (2013) that

$$
\mathrm{BB}\left(\mathcal{F}_{\omega}, Z\right)=\operatorname{Res}_{t=0}\left[\frac{\left(g_{1}(t) g_{2}(t)\right)^{2} d t}{g_{1}(t)}\right]=\operatorname{Res}_{t=0}\left[g_{1}(t)\left(g_{2}(t)\right)^{2}\right] .
$$

Since $g_{1}(y)\left(g_{2}(y)\right)^{2}$ is holomorphic at $y=0$, we get $\operatorname{BB}\left(\mathcal{F}_{\omega}, Z\right)=0$. In the third case $\mathcal{F}_{\omega}$ has a holomorhic first integral in neighborhood of $p$ and is known that $\operatorname{BB}\left(\mathcal{F}_{\omega}, Z\right)=0$.

The Baum-Bott formula implies the following result.

Corollary 3.4. Let $\omega \in \mathcal{F}(n, d), n \geq 3$, be a foliation with $K(\omega)=\emptyset$. Then there exists a smooth point $p \in S_{2}$ such that $j_{p}^{1} \omega=0$.

Proof. If for all smooth point $p \in S_{2}$ one has $j_{p}^{1} \omega \neq 0$, the above theorem shows that $\operatorname{BB}\left(\mathcal{F}_{\omega}, Z\right)=0$ for all irreducible components $Z \subset S_{2}$. By Baum-Bott's theorem, we get

$$
0<(d+2)^{2}=\sum_{Z \subset S_{2}} \operatorname{BB}\left(\mathcal{F}_{\omega}, Z\right) \operatorname{deg}[Z]=0
$$

which is a contradiction. Therefore there exists a smooth point $p \in S_{2}$ such that $j_{p}^{1} \omega=0$.

In particular, if $\omega \in \mathcal{F}(n, d), n \geq 3$, is a foliation with $j_{p}^{1} \omega \neq 0$ for any $p \in \mathbb{P}^{n}$, then its Kupka set is not empty.

\section{4 - THE NUMBER OF NON-KUPKA POINTS}

Through this section, we consider codimension one foliations on $\mathbb{P}^{3}$, but some results remain valid to codimension one foliations on others manifolds of dimension three. 


\section{1 - SIMPLE SINGULARITIES}

Let $\omega$ be a germ of 1 -form at $0 \in \mathbb{C}^{3}$. We define the rotational of $\omega$ as the unique vector field $\mathbf{X}$ such that

$$
\operatorname{rot}(\omega)=\mathbf{X} \Longleftrightarrow d \omega=\imath_{\mathbf{X}} d x \wedge d y \wedge d z
$$

moreover $\omega$ is integrable if and only if $\omega(\operatorname{rot}(\omega))=0$.

Let $\omega$ be a germ of an integrable 1 -form at $0 \in \mathbb{C}^{3}$. We say that 0 is a simple singularity of $\omega$ if $\omega(0)=0$ and either $d \omega(0) \neq 0$ or $d \omega$ has an isolated singularity at 0 . In the second case, these kind of singularities, are classified as follows

1. Logarithmic. The second jet $j_{0}^{2}(\omega) \neq 0$ and the linear part of $\mathbf{X}=\operatorname{rot}(\omega)$ at 0 has non zero eigenvalues.

2. Degenerated. The rotational has a zero eigenvalue, the other two are non zero and necessarily satisfies the relation $\lambda_{1}+\lambda_{2}=0$.

3. Nilpotent. The rotational vector field $\mathbf{X}$, is nilpotent as a derivation.

The structure near simple singularity is known (Calvo-Andrade et al. 2004). If $p \in S$ is a simple singularity and $d \omega(p)=0$, then $p$ is a singular point of $S$.

Theorem 4.1. Let $\omega \in \Omega^{1}\left(\mathbb{C}^{3}, 0\right), n \geq 3$, be a germ of integrable 1-form such that $\omega$ has a simple singularity at 0 then the tangent sheaf $\mathcal{F}=\operatorname{Ker}(\omega)$ is locally free at 0 and it is generated by $\langle\operatorname{rot}(\omega), \mathbf{S}\rangle$, where $\mathbf{S}$ has non zero linear part.

Proof. Let $\omega$ be a germ at $0 \in \mathbb{C}^{3}$ of an integrable 1 -form and 0 a simple non-Kupka singularity. Then $0 \in \mathbb{C}^{3}$ is an isolated singularity of $\mathbf{X}=\operatorname{rot}(\omega)$. Consider the Koszul complex of the vector field $\mathbf{X}$ at 0

$$
\mathbb{K}(\mathbf{X})_{0}: 0 \rightarrow \Omega_{\mathbb{C}^{3}, 0}^{3} \stackrel{\imath \mathbf{x}}{\longrightarrow} \Omega_{\mathbb{C}^{3}, 0}^{2} \stackrel{\imath \mathbf{X}}{\longrightarrow} \Omega_{\mathbb{C}^{3}, 0}^{1} \stackrel{\imath \mathbf{x}}{\longrightarrow} \mathcal{O}_{\mathbb{C}^{3}, 0} \rightarrow 0
$$

Since $\omega(\mathbf{X})=0$, then $\omega \in H^{1}\left(\mathbb{K}(\mathbf{X})_{0}\right)$ that vanishes because $\mathbf{X}$ has an isolated singularity at 0 . Therefore, there exists $\theta \in \Omega_{\mathbb{C}^{3}, 0}^{2}$ such that $\imath_{\mathbf{X}} \theta=\omega$. The map $\Theta_{\mathbb{C}^{3}, 0} \ni \mathbf{Z} \mapsto \imath_{\mathbf{Z}} d x \wedge d y \wedge d z \in \Omega_{\mathbb{C}^{3}, 0}^{2}$ is an isomorphism, hence

$$
\omega=\imath_{\mathbf{X}} \theta, \quad \text { and } \quad \theta=\imath_{\mathbf{S}} d x \wedge d y \wedge d z, \quad \text { implies } \quad \omega=\imath_{\mathbf{X}} \theta=\imath_{\mathbf{X}} \imath_{\mathbf{S}} d x \wedge d y \wedge d z
$$

and then, the vector fields $\{\mathbf{X}, \mathbf{S}\}$ generate the sheaf $\mathcal{F}$ in a neighborhood of 0 .

Let $\omega \in \mathcal{F}(3, d)$ be a foliation and $Z \subset S_{2}$ be a connected component of $S_{2}$. Assume that $Z$ is a local complete intersection and has only simple singularities. We will calculate the number $|N K(\omega) \cap Z|$ of non-Kupka points in $Z$.

Proof of Theorem 2. Let $\mathcal{J}$ be the ideal sheaf of $Z$. Since $Z$ is a local complete intersection, consider the exact sequence

$$
0 \rightarrow \mathcal{J} / \mathcal{J}^{2} \rightarrow \Omega^{1} \otimes \mathcal{O}_{Z} \rightarrow \Omega_{Z}^{1} \rightarrow 0
$$

Taking $\wedge^{2}$ and twisting by $L=K_{\mathbb{P}^{3}}^{-1} \otimes K_{Z}=K_{Z}(4)$ we get

$$
0 \rightarrow \wedge^{2} \mathcal{J} /\left.\mathcal{J}^{2} \otimes L \rightarrow \Omega_{\mathbb{P}^{3}}^{2}\right|_{Z} \otimes L \rightarrow \cdots
$$


Since $Z \subset S$, the singular set, we have seen before that

$$
\left.d \omega\right|_{Z} \in H^{0}\left(Z, \wedge^{2}\left(\mathcal{J} / \mathcal{J}^{2}\right) \otimes L\right)
$$

Now, from the equalities of sheaves

$$
K_{Z}^{-1} \otimes K_{\mathbb{P}^{3}} \simeq \wedge^{2}\left(\mathcal{J} / \mathcal{J}^{2}\right), \quad \text { and } \quad L \simeq K_{\mathbb{P}^{3}}^{-1} \otimes K_{\mathcal{F}}
$$

we have

$$
H^{0}\left(Z, \wedge^{2}\left(\mathcal{J} / \mathcal{J}^{2}\right) \otimes L\right)=H^{0}\left(Z,\left.K_{Z}^{-1} \otimes K_{\mathcal{F}}\right|_{Z}\right),
$$

the non-Kupka points of $\omega$ in $Z$ satisfies $\left.d \omega\right|_{Z}=0$, denoting

$$
D_{\omega}=\sum_{p \in Z} \operatorname{ord}_{p}(d \omega)
$$

the associated divisor to $\left.d \omega\right|_{Z}$, one has

$$
\operatorname{deg}\left(D_{\omega}\right)=\operatorname{deg}\left(K_{\mathcal{F}}\right)-\operatorname{deg}\left(K_{Z}\right)
$$

as claimed.

Remark 4.2. The method of the proof works also in projective manifolds, and does not depends on the integrability condition.

\section{2 - EXAMPLES}

We apply Theorem 2 for some codimension one holomorphic foliations on $\mathbb{P}^{3}$ and determine the number of non-Kupka points.

Example 4.3 (Degree two logarithmic foliations). Recall that the canonical bundle of a degree two foliation of $\mathbb{P}^{3}$ is trivial. There are two irreducible components of logarithmic foliations in the space of foliations of $\mathbb{P}^{3}$ of degree two: $\mathcal{L}(1,1,2)$ and $\mathcal{L}(1,1,1,1)$. We analyze generic foliations on each component.

Component $\mathcal{L}(1,1,2)$ : let $\omega$ be a generic element of $\mathcal{L}(1,1,2)$ and consider its singular scheme $S=S_{2} \cup S_{3}$. By Theorem 3 of Cukierman et al. (2006), we have $\ell\left(S_{3}\right)=2$. On the other hand, $S_{2}$ has three irreducible components, two quadratics and a line, the arithmetic genus is $p_{a}\left(S_{2}\right)=2$. Note that Theorem 2, implies that the number $\left|N K(\omega) \cap S_{2}\right|$, of non-Kupka points in $S_{2}$ is

$$
\left|N K(\omega) \cap S_{2}\right|=\operatorname{deg}\left(D_{\omega}\right)=\operatorname{deg}\left(K_{\mathcal{F}}\right)-\operatorname{deg}\left(K_{S_{2}}\right)=-\chi\left(S_{2}\right)=2 .
$$

The non-Kupka points of the foliation $\mathcal{F}_{\omega}$ are $|N K(\omega)|=\ell\left(S_{3}\right)+\left|N K \cap S_{2}\right|=4$.

Component $\mathcal{L}(1,1,1,1)$ : let $\omega$ be a generic element of $\mathcal{L}(1,1,1,1)$ then the tangent sheaf is $\mathcal{O} \oplus \mathcal{O}$ and the singular scheme $S=S_{2}$ (Giraldo and Pan-Collantes 2010), moreover consists of 6 lines given the edges of a tetrahedron, obtained by intersecting any two of the four invariant hyperplanes $H_{i}$. The arithmetic genus is $p_{a}\left(S_{2}\right)=3$, by Theorem 2, $|N K(\omega)|=\left|N K(\omega) \cap S_{2}\right|=4$, corresponding to the vertices of the tetrahedron where there are simple singularities of logarithmic type. 
Example 4.4 (The exceptional component $\mathcal{E}(3)$ ). The leaves of a generic foliation $\omega \in \mathcal{E}(3) \subset \mathcal{F}(3,2)$, are the orbits of an action of $\mathbf{A f f}(\mathbb{C}) \times \mathbb{P}^{3} \rightarrow \mathbb{P}^{3}$ and its tangent sheaf is $\mathcal{O} \oplus \mathcal{O}$ (see Calvo-Andrade et al. 2004, Giraldo and Pan-Collantes 2010). Its singular locus $S=S_{2}$ has $\operatorname{deg}(S)=6$ and three irreducible components: a line $L$, a conic $C$ tangent to $L$ at a point $p$, and a twisted cubic $\Gamma$ with $L$ as an inflection line at $p$. Then $N K(\omega)=L \cap C \cap \Gamma=\{p\} \subset S$.

The arithmetic genus is $p_{a}(S)=3$ and the canonical bundle of the foliation again is trivial, by Theorem 2 , the number of non-Kupka points $|N K(\omega)|=4$. Therefore the non-Kupka divisor $N K(\omega) \cap S=4 p$. If $\omega$ represents the foliation at $p$, then $\mu(d \omega, p)=\mu(\operatorname{rot}(\omega), p)=4$.

\section{ACKNOWLEDGMENTS}

The first author was partially supported by Fundação de Amparo à Pesquisa do Estado de São Paulo (FAPESP N N $^{\circ}$ 2014/23594-6), CONACYT 262121 and thanks the Federal University of Minas Gerais (UFMG), Instituto de Matemática Pura e Aplicada (IMPA), and Instituto de Matemática, Estatística e Computação Científica da UNICAMP for the hospitality during the elaboration of this work. The second author was partially supported by Coordenação de Aperfeiçoamento de Pessoal de Nível Superior (CAPESDGU 247/11), Conselho Nacional de Desenvolvimento Científico e Tecnológico (CNPq 300351/2012-3 and PPM-00169-13). The third author was partially supported by Bolsista/CAPES and thanks the Instituto de Matemática y Ciencias Afines (IMCA) for the hospitality. Finally, we would like to thank the referee by the suggestions, comments and improvements to the exposition.

\section{REFERENCES}

BAUM P AND BOTT R. 1972. Singularities of holomorphic foliations. J Differential Geometry 7: 279-342.

BRUNELLA M. 2009. Sur les Feuilletages de l'espace projectif ayant une composante de Kupka. Enseig Math (2) 55(3-4): $227-234$. BRUNELLA M AND PERRONE C. 2011. Exceptional singularities of codimension one holomorphic foliations. Publ Mathemàtique 55: 295-312.

CALVO-ANDRADE O. 1999. Foliations with a Kupka Component on Algebraic Manifolds. Bull of the Brazilian Math Soc 30(2): 183-197.

CALVO-ANDRADE O. 2016. Foliations with a radial Kupka set on projective spaces. Bull of the Brazilian Math Soc, 13 p. doi 10.1007/s00574-016-0158-6

CALVO-ANDRADE O AND SOARES M. 1994. Chern numbers of a Kupka component. Ann Inst Fourier 44: $1219-1236$.

CALVO-ANDRADE O, CERVEAU D, GIRALDO L AND LINS NETO A. 2004. Irreducible components of the space of foliations associated with the affine Lie algebra. Ergodic Theory and Dyn Sist 24: 987-1014.

CERVEAU D AND LINS NETO A. 1994. Codimension one Foliations in $\mathrm{Cp}^{\mathrm{n}}, \mathrm{n} \geq 3$, with Kupka components. In: Lins A, Moussu R and Sad P (Eds), Complex Analytic Methods in Dynamical Systems. Camacho C, Astérisque 222, p. 93-133.

CERVEAU D AND LINS NETO A. 2013. A structural theorem for codimension-one foliations on $\mathrm{P}^{\mathrm{n}}, \mathrm{n} \geq 3$, with application to degree three foliations. Ann Sc Norm Super Pisa Cl Sci (5) 12(1): 1-41.

CERVEAU D AND MATTEI JF. 1982. Formes intégrables holomorphes singulières. Astérisque 97, Paris: Soc Math de France.

CUKIERMAN F, SOARES M AND VAINSENCHER I. 2006. Singularities of Logarithmic foliations. Compositio Math 142: 131-142.

DE MEDEIROS A. 1977. Structural stability of integrable differential forms. Palis J and do Carmo M (Eds), Geometry and Topology, Springer LNM 597, p. 395-428.

GIRALDO L AND PAN-COLLANTES AJ.. 2010. On the singular scheme of codimension one holomorphic foliations in $\mathrm{P}^{3}$. Int $\mathrm{J}$ Math 21(7): 843-858.

GRIFFITHS PH AND HARRIS J. 1978. Principles of Algebraic Geometry. In Pure \& Appl Math, Wiley Interscience.

KUPKA I. 1964. Singularities of structurally stable Pfaffian forms. Proc Nat Acad of Sc USA 52: 1431-1432.

LORAY F. 2006. A preparation theorem for codimension-one foliations. Ann of Math (2) 163(2): 709-722. 
MALGRANGE B. 1976. Frobenius avec singularités. Codimension 1. IHES Publ Math 46: 163-173.

OKONEK CH, SCHNEIDER M AND SPINDLER H. 1980. Vector Bundles on Complex Projective spaces. Progress in Math Vol 3, Boston: Birkhauser, vii +389 p.

\section{5 - APPENDIX}

We prove Lemma 2.5 .

Proof. First, we see that $h^{0}(F) \geq 1$. By Riemann-Roch-Hirzebruch, we have

$$
\chi(F)=h^{0}(F)-h^{1}(F)+h^{2}(F)=\left[c h(F) \cdot T d\left(\mathbb{P}^{2}\right)\right]_{2}=2,
$$

then

$$
h^{0}(F)+h^{2}(F)=\left[\operatorname{ch}(F) \cdot T d\left(\mathbb{P}^{2}\right)\right]_{2}+h^{1}(F) \geq\left[\operatorname{ch}(F) \cdot T d\left(\mathbb{P}^{2}\right)\right]_{2}=2
$$

By Serre duality (Griffiths and Harris 1978, Okonek et al. 1980), we get $h^{2}(F)=h^{0}(F(-3))$. Moreover $h^{0}(F) \geq h^{0}(F(-k))$ for all $k>0$, hence $h^{0}(F) \geq 1$. Let $\tau \in H^{0}(F)$ be a non zero section, consider the exact sequence

$$
0 \longrightarrow \mathcal{O} \stackrel{\cdot \tau}{\longrightarrow} F \longrightarrow \mathcal{Q} \longrightarrow 0 \quad \text { with } \quad \mathcal{Q}=F / \mathcal{O} .
$$

The sheaf $\mathcal{Q}$ is torsion free, therefore $\mathcal{Q} \simeq \mathcal{J}_{\Sigma}$ for some $\Sigma \subset \mathbb{P}^{2}$. The sequence (5.1), is a free resolution of the sheaf $\mathcal{Q}$ with vector bundles with zero Chern classes. From the definition of Chern classes for coherent sheaves (Baum and Bott 1972), we get $c(\mathcal{Q})=1$, in particular $\operatorname{deg}(\Sigma)=c_{2}(\mathcal{Q})=0$, we conclude that $\Sigma=\emptyset$ and $\mathcal{Q} \simeq \mathcal{O}$. Then $F$ is an extension of holomorphic line bundles, hence it splits (Okonek et al. 1980, p. 15). 